\title{
Finger Flexion Deformity and Carpal Tunnel Syndrome Caused by Gouty Tophus
}

VARINDER KUMAR, MD, Rheumatology; CRAIG RODNER, MD, Associate Professor, Orthopedic Surgery; SANTHANAM LAKSHMINARAYANAN, MD, Associate Professor, Rheumatology, University of Connecticut School of Medicine, Farmington, Connecticut, USA. Address correspondence to Dr. V. Kumar, University of Connecticut School of Medicine, Rheumatology, 263 Farmington Ave., MC 5353, Farmington, Connecticut 06030, USA. E-mail: varinder.kumar@gmail.com. J Rheumatol 2015;42:1530-1; doi:10.3899/jrheum.150214

Although unusual, tophi in the absence of prior episodes of gouty arthritis can be the initial manifestation of gout ${ }^{1}$. Atypical triggering at the wrist and carpal tunnel syndrome because of intratendinous infiltration of tophaceous gout have been described only in older patients ${ }^{2,3}$.

A 20-year-old man was referred for the inability to extend the middle and ring fingers of the right hand, which was getting progressively worse. He also complained of tingling and numbness of fingers in median nerve distribution. On examination, he had no visible but only palpable volar mass at the right wrist. He appeared to have flexion contracture at the proximal interphalangeal (PIP) joints of the middle and ring fingers (Figure 1). Investigation revealed a serum uric acid level of $14.6 \mathrm{mg} / \mathrm{dl}$. Magnetic resonance imaging (MRI) of the right wrist (Figure 2) revealed a mass in the flexor digitorum superficialis (FDS) tendon sheath beginning just proximal to and extending into the carpal tunnel, and also with mass effect on adjacent median nerve. It was suggestive of a soft tissue mass, such as tendon sheath tumor or gouty tophus because both can have a similar appearance on $\mathrm{MRI}^{4}$. He required surgical exploration and excision of the FDS mass. Intraoperative diagnosis of gouty tophus was made because of the appearance (Figure 3), which was later confirmed on pathology. The main part of the gouty tophus existed proximal to carpal tunnel, preventing the tendon from moving under the flexor retinaculum, and therefore causing the fingers to have loss of extension, falsely giving the appearance of flexion contracture at the PIP joints. Postoperatively, he regained full extension and flexion and resolution of symptoms of median nerve compression.

Gouty tophus should be considered in male patients of any age who present with a mass within a tendon. Early diagnosis and treatment can lead to improved clinical outcomes.

\section{REFERENCES}

1. Wernick R, Winkler C, Campbell S. Tophi as the initial manifestation of gout. Report of six cases and review of the literature. Arch Intern Med 1992;152:873-6.

2. Sano K, Kohakura Y, Kimura K, Ozeki S. Atypical triggering at the wrist due to intratendinous infiltration of tophaceous gout. Hand 2009;4:78-80.

3. Mackford BJ, Kincaid RJ, Mackay I. Carpal tunnel syndrome secondary to intratendinous infiltration by tophaceous gout. Scand J Plast Reconstr Surg Hand Surg 2003;37:186-7.

4. Girish G, Melville DM, Kaeley GS, Brandon CJ, Goyal JR, Jacobson JA, et al. Imaging appearances in gout. Arthritis 2013;2013:673401

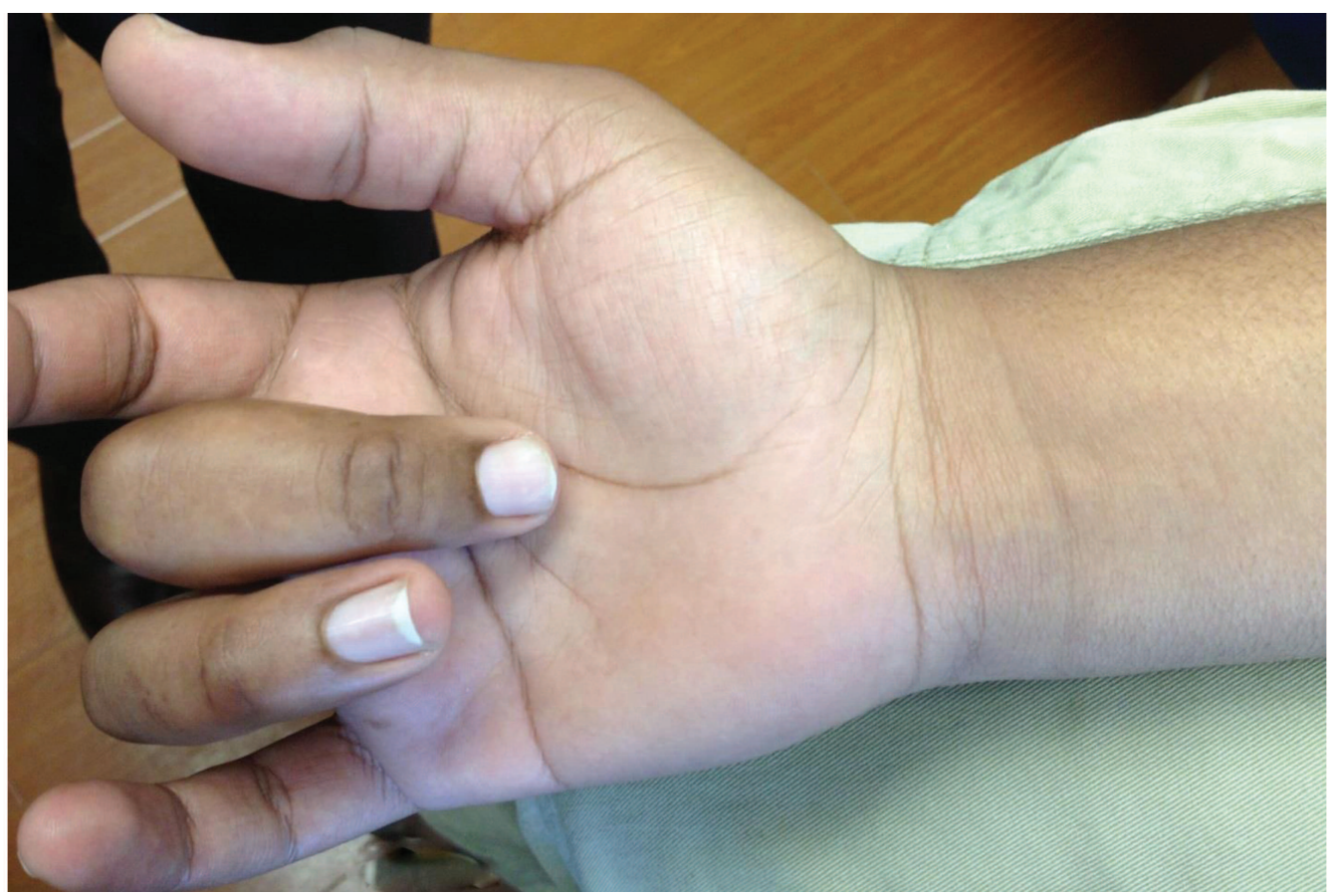

Figure 1. Initial presentation with flexion deformities at proximal interphalangeal joints of middle and ring fingers. 


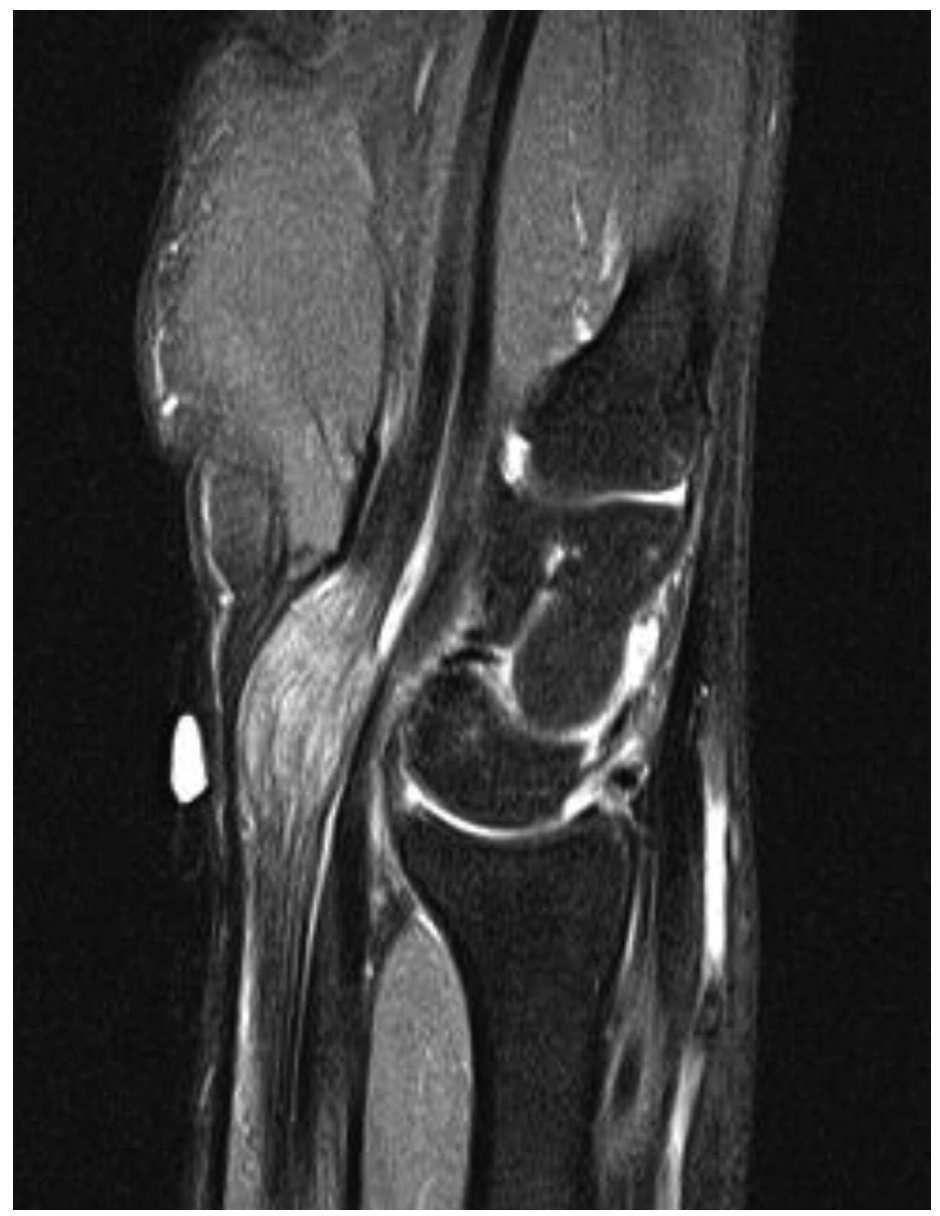

Figure 2. Proton-density fat-saturated magnetic resonance imaging of wrist shows mixed signal mass in flexor digitorum superficialis tendon.

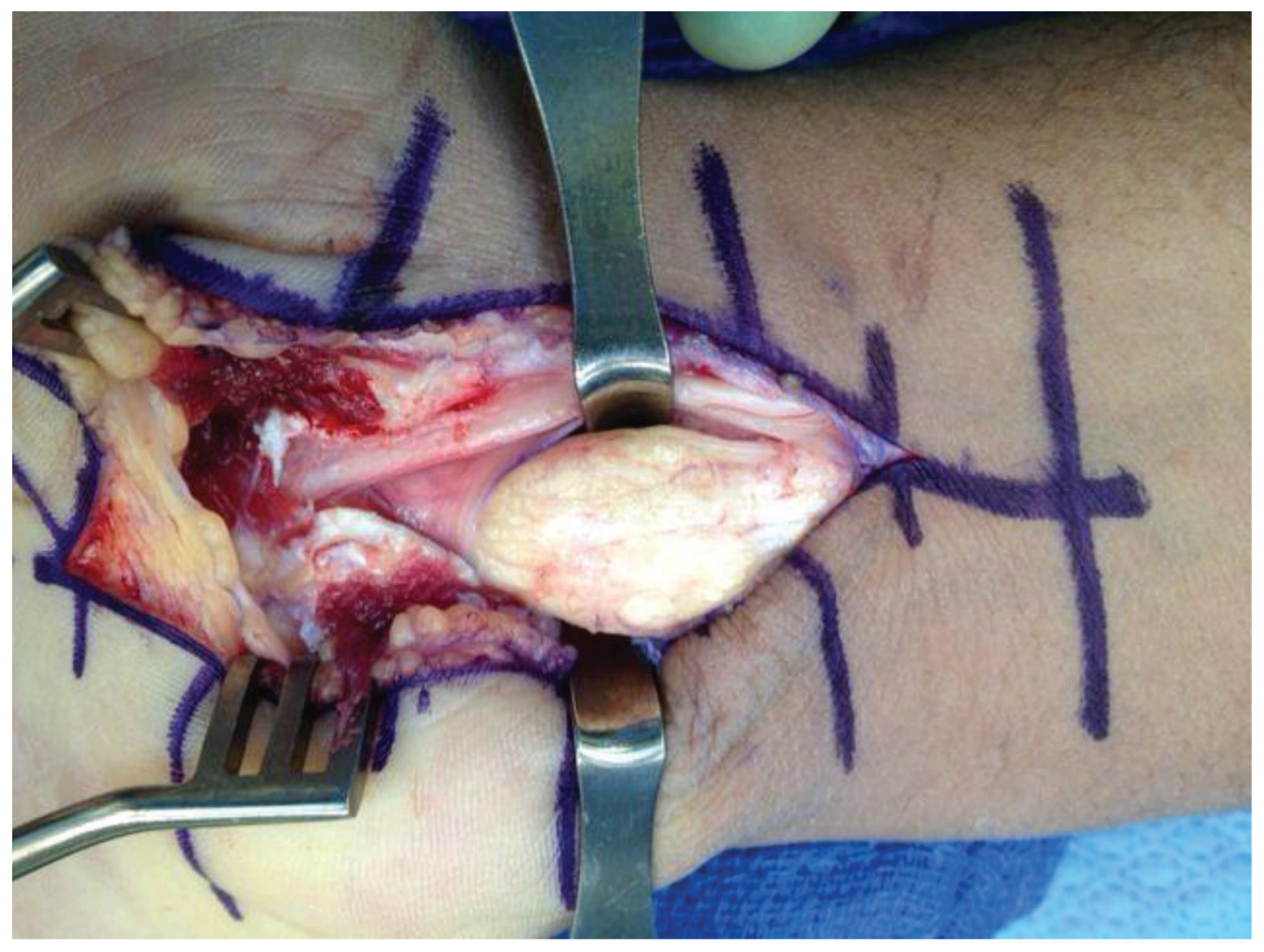

Figure 3. Intraoperative finding of gouty tophus in flexor digitorum superficialis tendon sheath. 